Bol. Acad. peru. leng. 63. 2018 (185-204)

\title{
ESTUDIO LÉXICO DEL GARÍFUNA: SINONIMIA Y PRÉSTAMOS LÉXICOS
}

\author{
LEXICAL STUDY OF GARIFUNA: \\ SYNONYMY AND LEXICAL BORROWINGS
}

María Trinidad Sánchez Pineda

\section{Resumen:}

Para este estudio se tomaron en cuenta dos campos léxicos de la lengua garífuna: flora y fauna. Se utilizó una metodología cuantitativa y cualitativa. La muestra fue de diez informantes, todos eran mayores de treinta años. En vista de que se pretendía obtener sinónimos y préstamos léxicos de nombres de plantas y animales, se realizaron preguntas utilizando un vocabulario determinado. En los resultados se evidenció la preponderancia de hispanismos en la lengua garífuna.

\section{Abstract:}

Two lexical fields of the Garifuna language were taken into account for this study: flora and fauna. Both quantitative and qualitative methodologies were used. The sample consisted of ten informants, all of them older than thirty. As the intention was to obtain synonyms and lexical borrowings of names of plants and animals, we asked questions using a certain vocabulary. The results showed the preponderance of Hispanisms in the Garifuna language. 
Palabras clave: lengua garífuna; sinonimia; préstamos léxicos.

Key words: Garifuna language; synonymy; lexical borrowings.

Fecha de recepción:

Fecha de aceptación:
$15 / 03 / 2018$

$31 / 05 / 2018$

\section{Introducción}

El garífuna es una de las lenguas habladas en Honduras por algunos afrodescendientes ${ }^{1}$. Ha tenido contacto lingüístico con el español, el inglés y el francés. Actualmente, está más de cerca con el español, puesto que es el idioma oficial de esta nación. En sus orígenes, también convivió con el arawak y el caribe.

En una entrevista realizada al antropólogo Andoni Castillo², miembro de Iriona, una de las comunidades garífunas, se comprueba que la lengua de los negros caribes ${ }^{3}$, en Honduras, es hablada en pocas zonas garífunas.

La lengua garífuna no se habla en un $100 \%$ en todas las comunidades garífunas. En Iriona y Gracias a Dios (es hablada de doce a trece comunidades) sí se habla en su totalidad. En Atlántida solo hay una comunidad en la que es hablada en un $90 \%$ y se trata de El Triunfo de la Cruz; del mismo modo, en la comunidad de Bajamar del departamento de Cortés. En el resto de las comunidades, hay tendencias del 20 al $40 \%$ de habla garífuna. En otras palabras, hay variaciones entre un departamento y otro.

1 El término se usa para sustituir al vocablo negros.

2 La entrevista se le hizo al informante en el año 2015.

3 Negros caribes: otra denominación que reciben los garífunas. 
https://doi.org/10.46744/bapl.201801.007

Honduras tiene dieciocho departamentos y los garífunas están concentrados en Cortés, Atlántida, Colón y Gracias a Dios. Sin embargo, en estos también hay personas mestizas o pertenecientes a pueblos indígenas. Lo notorio de esta situación ocurre cuando se indaga la vitalidad de la lengua garífuna y se concluye que los afrodescendientes prefieren aprender inglés o español que su lengua nativa. En consecuencia, son pocos quienes dominan la lengua garífuna, ya sea por razones de prestigio o de comercialización. Aunque estos factores no atañan directamente al estudio que aquí se presenta, se consideró necesario mencionarlos porque facilitan la comprensión del mismo.

En cuanto a las obras e investigaciones realizadas en la lengua garífuna se encuentran los diccionarios elaborados por Fernando Sabio, Alina Gómez y Salvador Suazo. Asimismo, la gramática de dicha lengua por Juan Diego Quesada, lingüista costarricense. En la actualidad, la Universidad Nacional Autónoma de Honduras a través de GRILIHO (Grupo de Investigación de Lenguas Indígenas de Honduras) está trabajando en un proyecto denominado Laboratorio garífuna que consiste en la impartición de cursos sobre cultura y lengua garífuna.

Sumado a lo anterior, se ofrece en este trabajo un estudio sobre sinónimos y préstamos del léxico de flora y fauna garífuna, lengua hablaba en el litoral Atlántico de Honduras. Se muestran algunas consecuencias del contacto lingüístico que involucran fenómenos en los diferentes niveles de la lengua, sobre todo, en el nivel léxico. Además, el estudio en cuestión permitirá a otros lingüistas la realización de nuevas investigaciones tomando en cuenta la metodología y algunos aspectos utilizados en esta.

Esta investigación será un aporte más para la lengua y la cultura garífuna. Además, de alguna forma, constatará que la influencia de las lenguas ocurre al existir un contacto entre ellas. También, que el nivel de lengua más afectado por dicho contacto es el léxico. En tal sentido, los préstamos léxicos son un fenómeno lingüístico que surgen de la influencia cultural, esta, a su vez, provocada por la lengua de la cultura que corresponda. 
Se ha indicado que la lengua garífuna ha tenido contacto con otras lenguas. No obstante, se piensa que la lengua española es la que más ha influido en esta, debido a su cercanía en los años anteriores y también en la actualidad. Además, es la segunda lengua (L2) de los garífunaparlantes.

Por tal razón, en el análisis de resultados se descubre si realmente en el léxico de flora y fauna del garífuna predomina los préstamos del español. O bien, si este presenta un porcentaje más alto de palabras prestadas de otros idiomas como el inglés o el francés.

\section{Metodología}

El modo de investigación utilizado para llevar a cabo este trabajo fue mixto (bibliográfico y de campo). Se consultaron fuentes escritas y digitales, además, se realizó un viaje al municipio de Iriona, departamento de Colón, con una duración de diez días. En ese lugar se visitaron dos comunidades garífunas: Iriona Viejo y Cusuna.

En el estudio que se llevó a cabo se tomaron en cuenta los aportes de diez afrodescendientes: cinco de Iriona Viejo y cinco de Cusuna. Con los últimos se realizó una reunión en la que todos contribuyeron a la investigación. No se determinó un rango de edad, por el contrario, se entrevistaron a personas con diferentes edades y sexo. Sin embargo, todos los informantes eran mayores de treinta años. De esa manera, todos los entrevistados podrían tener más dominio de su cultura y, en consecuencia, de su lengua.

El cuestionario utilizado para la recopilación de datos era sencillo. Se tenían los nombres en español de plantas y animales (vocabulario selecto). Después se formularon una serie de preguntas utilizando cada uno de estos nombres. Las interrogantes eran del tipo: ¿Cómo se dice gallina en lengua garífuna? Al obtener la respuesta, se procedía a realizar otra pregunta: ¿Utiliza en la lengua garífuna otro nombre para referirse a este animal? De esta forma, se encontró la diversidad de sinónimos. 
En el análisis del corpus léxico, se tomará en cuenta aspectos como los sinónimos, el posible origen de los términos y la realización fonética de estos. En el primero, se registrarán los términos que designan o refieren a un mismo elemento; en el segundo, a partir de los vocablos sinónimos se determinará el grado de competencia que se produce entre términos garífunas y préstamos.

Para esta investigación, se toma en cuenta el modelo mixto, ya que «representa el más alto grado de nivelación o combinación entre los enfoques cualitativo y cuantitativo. Ambos se entremezclan o combinan en todo el proceso de investigación o, al menos, en la mayoría de sus etapas. Agrega complejidad al diseño de estudio, pero contempla todas las ventajas de cada uno de los enfoques» (Hernández Sampiere 2003:21).

\section{Resultados}

\section{a. Campo semántico: flora Subcampo: plantas medicinales}

\begin{tabular}{|c|c|c|c|c|c|c|c|c|}
\hline$\ddot{Z}$ & 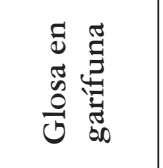 & 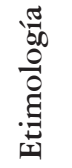 & 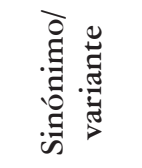 & 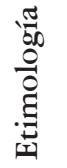 & 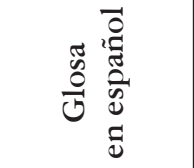 & 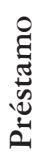 & 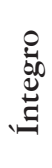 & 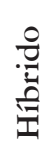 \\
\hline 1 & pipina & & papina & & calaica & & & \\
\hline 2 & pata & & & & nopal & & & \\
\hline 3 & guayui & & & & & & & \\
\hline 4 & lila hachuru & & Lila hachú & & pimienta gorda & & & \\
\hline 5 & fuesu & & & & pimienta & & & \\
\hline 6 & basen & ING & $\begin{array}{c}\text { helbak/ } \\
\text { alabahaca }\end{array}$ & ESP & albahaca & 3 & 1 & 2 \\
\hline 7 & carau & ESP & & & carao & 1 & & 1 \\
\hline 8 & misigadu & & & & nuez moscada & & & \\
\hline
\end{tabular}


María Trinidad Sánchez Pineda

https://doi.org/10.46744/bapl.201801.007

\begin{tabular}{|c|c|c|c|c|c|c|c|}
\hline 9 & masaniya & ESP & & manzanilla & 1 & & 1 \\
\hline 10 & romeu & ESP & & romero & 1 & & 1 \\
\hline 11 & hagüraha & & lemuru & & & & \\
\hline 12 & hasihasi & & & & & & \\
\hline 13 & flor de muerto & & & flor de muerto & 1 & 1 & \\
\hline 14 & guyâni & & & $\begin{array}{l}\text { mano de } \\
\text { lagarto }\end{array}$ & & & \\
\hline 15 & caleifa & & & hoja de aire & & & \\
\hline 16 & $\mathrm{Ti}$ & ESP & & zacate té & 1 & & 1 \\
\hline 17 & baleriánu & ESP & Taunligiali & valeriana & 1 & & 1 \\
\hline 18 & chichanbara & & & jengibre & & & \\
\hline 19 & ubuyibe & & & & & & \\
\hline 20 & gigímula & & kikímula & chichimora & & & \\
\hline 21 & tiliagu & & & cola de alacrán & & & \\
\hline 22 & lai & & & ajo & & & \\
\hline 23 & Kanela & ESP & & canela & 1 & & 1 \\
\hline 24 & wayu & & & & & & \\
\hline
\end{tabular}

Subcampo: plantas frutales

\begin{tabular}{|c|c|c|c|c|c|c|c|c|}
\hline Z & 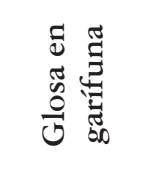 & 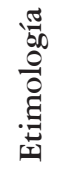 & ○ & 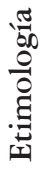 & 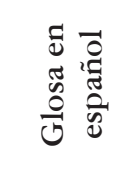 & 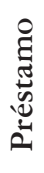 & : & $\begin{array}{l}\frac{0}{0} \\
\frac{0}{n=} \\
=\end{array}$ \\
\hline 1 & gain & & & & yuca & & & \\
\hline 2 & gumanana & & & & yuca dulce & & & \\
\hline 3 & guchu & & & & ñampán & & & \\
\hline 4 & yami & ESP & & & ñame & 1 & & 1 \\
\hline 5 & baruru & & & & plátano & & & \\
\hline
\end{tabular}




\begin{tabular}{|c|c|c|c|c|c|c|c|c|}
\hline 6 & gagani & & & & $\begin{array}{l}\text { plátano } \\
\text { hembra }\end{array}$ & & & \\
\hline 7 & uri & & & & marañón & & & \\
\hline 8 & aransu & ING & & & naranja & 1 & & 1 \\
\hline 9 & gurúsula & ESP & & & guanábana & 1 & 1 & \\
\hline 10 & singuela & ESP & & & Ciruela & 1 & & 1 \\
\hline 11 & fáluma & & & & Coco & & & \\
\hline 12 & guguedi & & & & $\begin{array}{l}\text { coco tierno } \\
\text { (de agua) }\end{array}$ & & & \\
\hline 13 & wáyafa & ESP & waríafa & ESP & guayaba & 2 & & 2 \\
\hline 14 & calip & ESP & eucalipto & ESP & eucalipto & 2 & 1 & 1 \\
\hline 15 & beibei & & baibei & & uva silvestre & & & \\
\hline 16 & bímina & & Fakudia & & banano & & & \\
\hline 17 & mângu & ESP & & & mango & 1 & & 1 \\
\hline 18 & sindurun & & sinduru & & limón & & & \\
\hline 19 & cherigi & & toonha & ESP & toronja & 1 & & 1 \\
\hline 20 & papamentu & & yérbabuena & ESP & hierbabuena & 1 & & 1 \\
\hline 21 & ababaü & & & & papaya & & & \\
\hline 22 & murei & & & & nance & & & \\
\hline 23 & yeyagua & & & & piña & & & \\
\hline 24 & mângusapatu & ESP & & & $\begin{array}{l}\text { mango } \\
\text { zapato }\end{array}$ & 1 & & 1 \\
\hline 25 & awasi & & & & maíz & & & \\
\hline 26 & uludi & ESP & iludi & ESP & elote & 2 & & 2 \\
\hline 27 & mapi & & mabi & & camote & & & \\
\hline 28 & wagadi & ESP & & & aguacate & 1 & & 1 \\
\hline 29 & bariñe & & culantro & ESP & culantro & 1 & 1 & \\
\hline 30 & higagu & ESP & & & hicaco & 1 & & 1 \\
\hline 31 & guribiyua & & & & coyol & & & \\
\hline 32 & almendra & & & & almendra & 1 & & 1 \\
\hline 33 & mansana & ESP & masana & ESP & manzana & 2 & & 2 \\
\hline
\end{tabular}


María Trinidad Sánchez Pineda

https://doi.org/10.46744/bapl.201801.007

\begin{tabular}{|c|c|c|c|c|c|c|c|c|}
\hline 34 & badiya & ESP & badía & ESP & sandía & 2 & & 2 \\
\hline 35 & gabu & & cacao & & cacao & 1 & & 1 \\
\hline 36 & góubana & & & & caoba & & & \\
\hline 37 & moringa & ESP & & & moringa & 1 & 1 & \\
\hline 38 & noni & ESP & & & noni & 1 & 1 & \\
\hline 39 & uréganu & ESP & & & orégano & 1 & & 1 \\
\hline 40 & uraco & & & & $\begin{array}{c}\text { zapote } \\
\text { grande }\end{array}$ & & & \\
\hline 41 & sabudi & ESP & & & zapote & 1 & & 1 \\
\hline 42 & mokikia & & mókinkiapu & & $\begin{array}{c}\text { Zapote } \\
\text { amarillo }\end{array}$ & & & \\
\hline 43 & laimani & & kahela & ESP & cangela & 1 & & 1 \\
\hline 44 & gûruba & & gurúa & & mangle & & & \\
\hline 45 & fulagun & & & & $\begin{array}{c}\text { mata de } \\
\text { guineo }\end{array}$ & & & \\
\hline
\end{tabular}

b. Campo semántico: fauna

Subcampo: animales acuáticos

\begin{tabular}{|c|c|c|c|c|c|c|c|c|}
\hline$\dot{Z}$ & 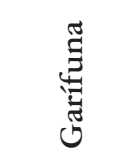 & 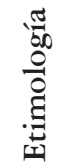 & 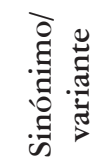 & 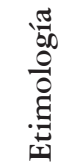 & 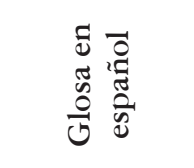 & 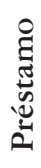 & : & 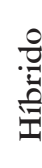 \\
\hline 1 & gadaru & & & & tortuga marina & & & \\
\hline 2 & gaguamu & ESP & & & cagüamo & 1 & & 1 \\
\hline 3 & garou & & & & carey & & & \\
\hline 4 & buldusu & & abadaru & ESP & apartado & 1 & & 1 \\
\hline 5 & udura & & uduraü & & pescado & & & \\
\hline 6 & gagali & ESP & & & pescado calale & 1 & & 1 \\
\hline 7 & gadabilua & & & & & & & \\
\hline 8 & hiyagua & & & & resnapa & & & \\
\hline 9 & awaguri & & kinfich & ING & quinfix & 1 & & 1 \\
\hline
\end{tabular}


ESTUDIO LÉXICO DEL GARIFUNA: SINONIMIA Y PRÉSTAMOS LÉXICOS https://doi.org/10.46744/bapl.201801.007

\begin{tabular}{|c|c|c|c|c|c|c|c|}
\hline 10 & gulilawarü & ESP & & pez culila & 1 & & 1 \\
\hline 11 & yaguaigau & & & curel & & & \\
\hline 12 & sabadelu & & & pez palometa & & & \\
\hline 13 & gifiyu & & & jurelito & & & \\
\hline 14 & gurisawa & & & sardina & & & \\
\hline 15 & sügüri & & sigei & pez tonina & & & \\
\hline 16 & gasíaran & & warübi/gasía & pez sierra & & & \\
\hline 17 & tilapia & ESP & & tilapia & 1 & 1 & \\
\hline 18 & gamarun & ESP & isuru & camarón & 1 & & 1 \\
\hline 19 & hüru & & & cangrejo & & & \\
\hline 20 & agare & & & lagarto & & & \\
\hline 21 & herenge & & & cangrejo de playa & & & \\
\hline 22 & guayumi & & gusa & cangrejos azules & & & \\
\hline 23 & bahü & & & cangrejo de río & & & \\
\hline 24 & harouru & & & jaiba & & & \\
\hline 25 & hamaba & & & jaiba & & & \\
\hline 26 & biribiri & & & jaiba & & & \\
\hline 27 & magüñü & & & jaiba & & & \\
\hline 28 & biritauba & & & róbalo & & & \\
\hline 29 & sáwalu & ESP & haba & sábalo & 1 & & 1 \\
\hline 30 & awachúchu & & & nutria & & & \\
\hline 31 & wéibayawa & & & tiburón & & & \\
\hline 32 & manadi & ESP & & manatí & 1 & & 1 \\
\hline 33 & yalifu & & & pelícano & & & \\
\hline 34 & sügüri & & & delfín & & & \\
\hline 35 & sáwawa & & sáwarawara & gaviota & & & \\
\hline
\end{tabular}


Subcampo: animales domésticos

\begin{tabular}{|c|c|c|c|c|c|c|c|c|}
\hline$\dot{Z}$ & 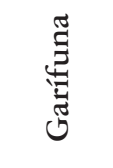 & 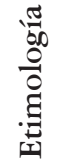 & 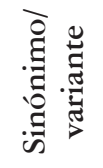 & 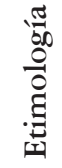 & 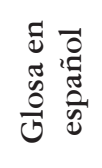 & 苞 & 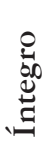 & $\begin{array}{l}\frac{0}{0} \\
\frac{0}{2} \\
=\end{array}$ \\
\hline 1 & arira & & gallu & ESP & gallina & 1 & & 1 \\
\hline 2 & aunly & & & & perro & & & \\
\hline 3 & bágasu & ESP & adabiu & & vaca & 1 & & 1 \\
\hline 4 & buíruhu & & & & cerdo & & & \\
\hline 5 & burihu & & & & cerdo & & & \\
\hline 6 & figaga & & & & jolote & & & \\
\hline 7 & ganaru & & patu & ESP & Pato & 1 & & 1 \\
\hline 8 & mesu & & & & gato & & & \\
\hline 9 & suafu & & $\begin{array}{l}\text { suáfuru } \\
\text { /gabáyu }\end{array}$ & ESP & caballo & 1 & & 1 \\
\hline
\end{tabular}

Subcampo: animales silvestres

\begin{tabular}{|c|c|c|c|c|c|c|c|c|}
\hline$\ddot{Z}$ & 胥 & 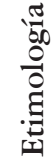 & 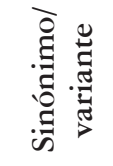 & 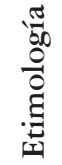 & 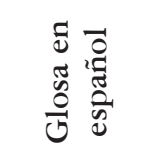 & 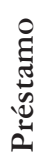 & : & 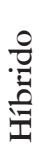 \\
\hline 1 & wayamaga & & & & iguana & & & \\
\hline 2 & benou & ESP & usari & & venado & 1 & & 1 \\
\hline 3 & gasigamu & & & & cusuco & & & \\
\hline 4 & bigibû & & & & ardilla & & & \\
\hline 5 & másaraga & & & & conejo & & & \\
\hline 6 & gurewegi & & lora & ESP & loro & 1 & & 1 \\
\hline 7 & erangunu & & mapachi & ESP & mapache & 1 & & 1 \\
\hline 8 & kekeo & & & & $\begin{array}{c}\text { chancho de } \\
\text { monte }\end{array}$ & & & \\
\hline
\end{tabular}


ESTUDIO LÉXICO DEL GARIFUNA: SINONIMIA Y PRÉSTAMOS LÉXICOS https://doi.org/10.46744/bapl.201801.007

\begin{tabular}{|c|c|c|c|c|c|c|c|c|}
\hline 9 & gaigusi & & & & tigre & & & \\
\hline 10 & danto & ESP & dandei & ESP & dante & 2 & & 2 \\
\hline 11 & gibinadu & & & & tepezcuintle & & & \\
\hline 12 & coyote & & & & coyote & 1 & 1 & \\
\hline 13 & musegalu & ESP & buriri & & murciélago & 1 & & 1 \\
\hline 14 & húa & & & & sapo & & & \\
\hline 15 & tumú & & dunuru & & pájaro & & & \\
\hline 16 & héweraü & & & & gusano & & & \\
\hline 17 & hewe & & & & culebra & & & \\
\hline 18 & lefan & & & & elefante & 1 & & 1 \\
\hline 19 & yurüdü & & $\begin{array}{l}\text { achuuhati } \\
\text { fuluri }\end{array}$ & & colibrí & & & \\
\hline 20 & garadun & & & & ratón & & & \\
\hline 21 & dagúasi & ESP & mótete & & tacuacín & 1 & & 1 \\
\hline 22 & gúnrere & & anasi & & araña & & & \\
\hline 23 & gubari & & & & garrapata & & & \\
\hline 24 & águru & & & & alacrán & & & \\
\hline 25 & liñún & ING & liun & ING & león & 2 & 1 & 1 \\
\hline 26 & yagüri & & yagüi & & oropéndola & & & \\
\hline 27 & gárarawa & ESP & & & guara roja & 1 & & 1 \\
\hline 28 & gáduri & & $\begin{array}{l}\text { duguyu/ } \\
\text { lechusa }\end{array}$ & ESP & lechuza & 1 & & 1 \\
\hline 29 & garún & & & & gavilán & & & \\
\hline 30 & gibidiliyau & & & & oso & & & \\
\hline 31 & asfina & & & & oso hormiguero & & & \\
\hline 32 & aguri & & & & guatusa & & & \\
\hline 33 & $\begin{array}{l}\text { dundun } \\
\text { wewe }\end{array}$ & & & & $\begin{array}{c}\text { pájaro } \\
\text { carpintero }\end{array}$ & & & \\
\hline 34 & üwi & & & & boa & & & \\
\hline
\end{tabular}




\begin{tabular}{|c|c|l|l|l|l|l|l|l|}
\hline 35 & black manu & & & & & & & \\
\hline 36 & awada & & & & $\begin{array}{c}\text { mono cara } \\
\text { blanca }\end{array}$ & & & \\
\hline
\end{tabular}

\section{Subcampo: insectos}

\begin{tabular}{|c|c|c|c|c|c|c|c|c|}
\hline$\dot{\mathrm{Z}}$ & 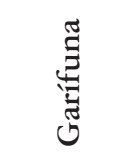 & 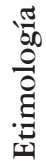 & 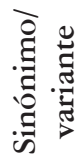 & 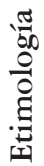 & 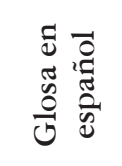 & 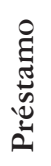 & 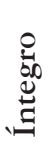 & 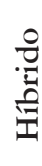 \\
\hline 1 & werewere & & & & mosca & & & \\
\hline 2 & hulahünü & & & & avispa & & & \\
\hline 3 & fudi & & & & cucaracha & & & \\
\hline 4 & seriseri & & & & zompopo & & & \\
\hline 5 & gunga & & & & tábano & & & \\
\hline
\end{tabular}

\section{Discusión}

Las tablas anteriores muestran que en la mayoría de los préstamos del español en la lengua garífuna se encuentra la terminación $u$ y $\ddot{u}$. En la adaptación de las palabras prestadas es constante este tipo de terminación. Y se verifica en los fenómenos encontrados en el corpus.

Hay vocablos que presentan tres variantes/sinónimos en garífuna: basen, belbak y albahaca son términos del garífuna utilizados para referirse aalbahaca. Esto indica que algunos parlantes de esa lengua utilizan el préstamo íntegro del español albabaca. En cambio, otros recurren abelbak, un préstamo híbrido, siempre del español. Cabe señalar que la mayoría de los informantes indican basen; palabra que en inglés equivale a basil.

Otro caso de sinonimia en los vocablos: hagürahaylemuru es un árbol que cura la sinusitis, pero no tiene un significante en español. Por 
https://doi.org/10.46744/bapl.201801.007

su fonética, se puede afirmar que ambos términos son de origen arawak. Hay que recordar que esta lengua (arawak) también tuvo contacto con la garífuna y que es inevitable su influencia, sea esta léxica, sintáctica o en cualquiera de los niveles lingüísticos.

Una planta medicinal muy conocida en la lengua garífuna es la chichimora, cuyo nombre tiene dos variantes: gigímulaykikimula. La diferencia entre estos dos términos radica en las consonantes de las dos primeras sílabas. Desde el punto de vista fonético se puede afirmar que hay un ensordecimiento de consonantes oclusivas: $/ \mathrm{g} />/ \mathrm{k} /$.

De acuerdo con lo investigado, hay dos formas de nombrar en el garífuna al banano: bímina y fakudia son palabras que no presentan grado de semejanza con la escritura del término en español, por tanto, se consideran de origen arawak.

Existen otros ejemplos de sinónimos en plantas comestibles y uno de ellos se identifica en la palabra toronja. En la lengua garífuna se le conoce como cherigi o toonha. Se aduce que estos términos son de origen distinto. El primero presenta características arawakas, por tanto, se afirma que procede de esta lengua. Y, el segundo es un préstamo híbrido del español. Lo último se asocia al nivel fonético, pues tanto toonha como toronja son muy similares en ese aspecto.

Las entradas papamentu y yérbabuena aparecen como sinónimos en garífuna y aluden a bierbabuena. A la segunda entrada se le denomina préstamo híbrido del español por su grado de semejanza con la escritura del término en esa lengua. En cambio, la primera tiene dos posibles orígenes: uno arawak y otro caribe. Se dan estas dos posibilidades tomando en cuenta que los garífunas tuvieron contacto con los grupos que hablaban estas lenguas.

En el garífuna, para referirse a culantro, hay dos ítems: bariñe y culantro. Es evidente que el segundo ítem es un préstamo íntegro del español porque no ha sufrido ningún cambio en su adaptación. Y el segundo tiene un origen arawak o caribe. Es importante indicar que la 
mayoría de los informantes dijeron culantro, muy pocos nombraron bariñe (casi solo las personas mayores de 70 años). Esto indica que el primer término no es muy utilizado por la población de edad temprana.

Para referirse al término cangela, algunos informantes dijeron laimani y otros, kabela. Prácticamente, el primer vocablo aportado es el menos usado y se cree que es de origen arawak o caribe. En cuanto al segundo, es un préstamo híbrido del español. Se afirma que pertenece a este tipo de préstamo porque en su adaptación se encuentra un parecido a la escritura de esta lengua, es decir, no está demostrando exactitud en su grafía ni en su fonética.

Además de las variantes anteriores, se encuentran sindurun y sinduru (limón en garífuna). Aquí se identifica la forma apocopada del primer término. Sin embargo, otros podrían afirmar que este caso es un metaplasmo de tipo paragoge, en vista de que no se sabe a ciencia cierta a qué término se elidió (o se agregó) el morfema.

Con mapiy mabi sucede el fenómeno de la sonorización de oclusivas. Es decir, la consonante oclusiva bilabial sorda /p/ se convierte en oclusiva bilabial sonora /b/. La discrepancia entre ellos es mínima, pero en la lengua garífuna los dos se usan para nombrar a camote.

Como se puede observar, uludi e iludi son semejantes, la diferencia radica en que la vocal inicial de la primera es posterior redondeada y de la segunda es anterior no redondeada. Sin embargo, los dos términos se utilizan para aludir a elote, término de la lengua española.

Además de los préstamos que ya se han expuesto, están mansana y masana. Es claro que son términos que proceden del español y fueron adaptados a la lengua garífuna tomando en cuenta su fonética; ya que el cambio entre el término del español (manzana) a la segunda ocurre en dos morfemas en (masana). Aquí se da elisión del fonema nasal alveolar sonoro $/ \mathrm{n} / \mathrm{y}$ su escritura ya no es con la consonante fricativa interdental sorda $/ \theta /$, sino con la fricativa alveolar sorda $/ \mathrm{s} /$; y uno en (mansana), en 
https://doi.org/10.46744/bapl.201801.007

este último sucede el intercambio de fonemas fricativos que se explicó anteriormente.

El término sandía tiene dos variantes en el idioma garífuna: badiya y badía. Ambos términos se originan del español. Por consiguiente se afirma que son préstamos híbridos de esa lengua. Por su parte gûruba y gurúa, términos que se utilizan para referirse a mangle, pueden ser de origen arawak o caribe.

Después de haber terminado la discusión de sinónimos/variantes y préstamos léxicos del vocabulario de la flora se procede al abordaje de estos fenómenos, pero en el campo léxico de la fauna. El primer ejemplo de sinonimia: buldusulabadaru (en español apartado), un tipo de pez de la especie de róbalo.

Es raro encontrar una palabra de origen inglés, pero en el siguiente caso awaguri/kinfich se ve claramente un anglicismo, y se trata del segundo elemento. Ambos términos se refieren al pescado quinfix, aún no se han registrado en una obra lexicográfica.

En gamarum/isuru (ambas lexicalizadas), su primer elemento es un préstamo híbrido del español, y su segundo se podría catalogar originario de la lengua arawak o, en todo caso, caribe. Estos sinónimos se utilizan para referirse a camarón, y lo que ocurre en la adaptación del préstamo es la sonorización de la oclusiva $/ \mathrm{k} /$, además de la terminación de su última sílaba en la vocal $u$.

El término ballena presenta tres vocablos en garíguna: udu/ballenal amana. Por el contrario, pescado, presenta dos: udura/uduraü. En este último caso, se identifica un tipo metaplasmo denominado apócope, ya que se le elimina la vocal ü de udura. No obstante, el más usado por los informantes es el primer elemento.

A su vez, gasíaran/gasía son similares tanto en su escritura como en su fonética, en español, pez sierra. El fenómeno lingüístico descubierto entre ambos es el denominado paragoge, se asume que pertenece a este 
tipo de metaplasmo porque la variante más repetida por los informantes fue gasía.

La diferencia entre sáwawa/sáwarawara y gaviota en la lengua española radica en la incorporación de elementos en medio y al final a la primera entrada. Por tanto, se encuentra un ejemplo de epéntesis y otro de paragoge, respectivamente. Los fenómenos lingüísticos encontrados se le adjudican a la segunda entrada porque la más mencionada es sáwawa.

Las palabras arira/gallu se refieren a gallina/gallo. Ambos son préstamos del español. Lo notable de dichas palabras ocurre en su uso, particularmente, las personas mayores de cincuenta años utilizan el primero y las personas más jóvenes, el segundo.

En cuanto a bagasuladabiu, vaca en español, son notables aspectos parecidos al ejemplo anterior. Esto es, la terminación en $u$ de la palabra prestada, en este caso, bagasu. Lo que ocurre con el préstamo es una adaptación fonética. Con respecto a suafu/gabáyu (caballo en la lengua española), se afirma que el primero es un arawakismo y el segundo un préstamo híbrido del español. Se ha señalado frecuentemente que la fonética desempeña un papel primordial en los préstamos, y se ve reflejado en gabayu: se da la sonorización de la primera consonante oclusiva y la asimilación de su última consonante, además de la terminación en $u$.

Los siguientes términos ganaru/patu se utilizan para nombrar a pato en el español. Para el término venado, se usan dos palabras en el idioma garífuna: benou/usari. En la primera se da una adaptación fonética del español al garífuna; asimismo, se resalta la terminación de la palabra en vocal $u$. La segunda es mencionada especialmente por los ancianos de la comunidad y, posiblemente, de origen arawak.

De igual manera, el grupo de sinónimos musegalulburiri, que hacen alusión a murciélago contiene una palabra de origen español, se trata del primer elemento. Se le considera un préstamo híbrido. Para referirse a colibrí, se usan los siguientes vocablos: yurüdül achuubatifuluri. Es importante recalcar que ninguno de ellos está registrado en un 
https://doi.org/10.46744/bapl.201801.007

diccionario; no obstante, el más frecuente entre los hablantes es yurüdü. Se aduce que su etimología no procede ni del español ni del inglés; más bien, es arawak o caribeña.

Posteriormente, en el grupo dagúasi/mótete se identifica un préstamo híbrido del español, ya que el primer elemento es muy parecido a tacuacín. Cabe señalar que dicho préstamo es el más nombrado por los informantes, a pesar de estar lexicalizadas las dos palabras. El étimo del vocablo restante posiblemente sea caribe o arawak.

Finalmente, es preciso indicar que en el léxico de flora y fauna de la lengua garífuna hay preponderancia de préstamos del español. Esto indica que la hipótesis de este trabajo es verdadera; es decir, de una presunción se obtuvo una aseveración.

En los campos léxicos estudiados de la lengua garífuna se encuentran muchos sinónimos. El inglés y el francés son idiomas que no han influido en gran medida en el léxico de animales y plantas de la lengua garífuna. Los hallazgos que se encontraron de estos son mínimos.

Algunos de los vocablos recopilados presentan una o más variantes. Es decir, se identifican fenómenos en los que dos o tres palabras se diferencian por una cantidad mínima de grafemas. Muchos de los términos presentan etimología dudosa. Esto indica que no se conoce con certeza el origen de los mismos.

En cuanto a la fauna, se encontró predominio de animales acuáticos en el corpus. Por tanto, se aduce que el mar es uno de los elementos importantes para la cultura garífuna, debido a la actividad de la pesca.

Con respecto a campo léxico de flora, se afirma que se encontró un porcentaje inesperado de plantas medicinales. Esto marca la dependencia que tienen los garinagu hacia la medicina natural.

El acceso a las comunidades donde se realizó la recolección de datos es un poco difícil, debido a que se requiere dos días para llegar al 
destino. Además, muchos kilómetros de la carretera no se encuentran en condiciones viables.

En los lugares visitados se imparten cinco horas semanales de lengua materna (en este caso, la garífuna); sin embargo, se considera que dichas horas no representan el tiempo suficiente para enseñar un idioma y contribuir a la preservación del mismo. 
https://doi.org/10.46744/bapl.201801.007

\section{BIBLIOGRAFÍA}

AMEIDA, M. (1999). Sociolingüística. Colección Materiales didácticos universitarios. La Laguna: Servicio de Publicaciones de la Universidad de La Laguna.

ANDRADE, R. G. de. (1981). Los negros caribes de Honduras. Tegucigalpa, Honduras: Guaymuras.

APPEL, R. y MUYSKEN, P. (1996). Bilingüismo y lenguas en contacto. Barcelona, España: Editorial Ariel.

CHUNLAN, W. (2004). El anglicismo en el léxico chino mandarín y en el léxico español: su incidencia en la enseñanza-aprendizaje de español como lengua extranjera. Facultad de Filología. Universidad Complutense de Madrid. Disponible en: http://biblioteca. ucm.es/tesis/fll/ucm-t28426.pdf. (Consultado el 15 de junio del 2015).

DAVIDSON, W. V. (2009). Etnología y etnohistoria de Honduras. Tegucigalpa, Honduras: Instituto Hondureño de Antropología e Historia.

GRAMÁTICA ESCOLAR GARÍFUNA (2002). Garudia lánina lafansehaun hererun garinagu. Impresora Comercial Ortíz. Secretaría de Educación, PRONEEAAH.

HERNÁNDEZ SAMPIERE, R. (2003). Metodología de la investigación. 3. ed. México: McGraw-Hill Interamericana.

L. GONZÁLEZ, N. (2008). Peregrinos del Caribe; etnogénesis y etnobistoria de los garífunas. Honduras: Guaymuras.

LARA PINTO, G. (2002). Perfil de los pueblos indígenas y negros de Honduras. Tegucigalpa, Honduras: Unidad Regional de Asistencia Técnica. 
https://doi.org/10.46744/bapl.201801.007

LAROUSSE. Diccionario Básico. (s. f.). Español/Francés. 11. a ed. México: Ediciones Larousse.

LÓPEZ MORALES, H. (1994). Métodos de Investigación Lingüística. Salamanca, España: Ediciones Colegio de España.

RAMOS, V. M. (2013). Diccionario de las lenguas de Honduras. Tegucigalpa, Honduras: Caracol Impresiones.

SABIO, F. y ORDOÑEZ, A. (2006). Hererun Wagüchagu. La Ceiba, Honduras: Asociación Misionera Garífuna.

SALA, M. (1988). Elproblema de las lenguas en contacto. México: Universidad Nacional Autónoma de México. D.F.

SECRETARÍA DE EDUCACIÓN, PRONEEAAH (2005). Furunde Wamonweñeñe. Aprendamos nuestra lengua. Colección cipotes de nuestros pueblos N. ${ }^{\circ} 1$ Primer grado.

SECRETARÍA DE EDUCACIÓN, PRONEEAAH (2003). Liwamutuburu wamouwañeñe. Escribimos y leemos nuestra lengua garífuna Colección cipotes N. ${ }^{\circ}$ 3. Tercer grado. Tegucigalpa, Honduras.

SUAZO, S. (2001). Lila Garifuna (Diccionario garífuna-garífuna español). Tegucigalpa, Honduras: Litografía López. 